\section{Gaze Palsy in Children: What is Underneath? - A Review Article}

\section{Abstract}

Eye movement abnormalities always clench the eyes of a neurologist to reach an interesting diagnosis most of the time in children. The common etiologies affecting the complex brainstem pathways and frontal eye field controlling conjugate eye movement are childhood stroke (pontine infarct), demyelinating disorders, mass lesions, trauma and metabolic or mitochondrial diseases. Horizontal eye movements are conducted by the medial rectus and the lateral rectus muscles. Medial rectus is innervated by oculomotor nerve (cranial nerve III) and the lateral rectus is innervated by abducens nerve (cranial nerve VI) respectively. The oculomotor and the abducens nuclei are interconnected by medial longitudinal fasciculus (MLF). The disorders of horizontal eye movement that are caused by brainstem lesions are classified into three groups: lateral gaze palsy, internuclear ophthalmoplegia, and one-and-a-half syndrome. In the present study, three interesting cases with gaze palsy have been taken into account. Case 1 is an 8-year-old boy presented with left sided hemiparesis and right sided gaze palsy with loss of adduction in right eye (One-and-half syndrome). MRI showed large areas of increased T2W signal intensity both in subcortical white matter and brain stem (involving abducens nucleus, PPRF and ipsilateral MLF). Case 2 is also of a 10-year-old boy, diagnosed to be a case of Clinically Isolated Syndrome (CIS) with ataxia and ophthalmoplegia. MRI showed areas of sub-cortical demyelination in both fronto-parietal region (right side more involved than left) with brain stem and cerebellum unaffected. Case 3 is about an 18-month-old boy presented with complete ophthalmoplegia (Inability to move both the eyes with absent conjugate movement in all directions) and delayed developmental milestones. Lactate was raised in venous blood and MRI showed necrosis in basal ganglia (thalami) and brain stem, with MR spectroscopy showed double lactate peak consistent with Leigh disease (mitochondrial encephalomyopathy).

Keywords: Ophthalmoplegia; Brainstem; One-and-half syndrome; Encephalomyopathy

\section{Jayitri Mazumdar', Sumita Pall, Gautam De ${ }^{1}$ and Kartik Chandra Ghosh ${ }^{2}$}

\author{
1 Department of Pediatrics, Calcutta \\ National Medical College and Hospital, \\ Kolkata, India \\ 2 Department of Neurology, Calcutta \\ National Medical College and Hospital, \\ Kolkata, India
}

\section{*Corresponding author: \\ Dr. Jyitri Mazumdar, M.D}

” jayidoc@gmail.com

Pediatrics, Senior Resident, Department of Pediatrics, Calcutta National Medical College and Hospital, Kolkata, India.

Tel: 09230297392

Citation: Mazumdar J, Pal S, De G, Ghosh KC (2017) Gaze Palsy in Children: What is Underneath? - A Review Article. J Neurol Neurosci. Vol. 8 No. 4:207

Received: June 24, 2017; Accepted: July 14, 2017; Published: July 18, 2017

\section{Introduction}

One-and-a-half syndrome occurs with lesions in the dorsal pontine tegmentum that impair both the ipsilateral PPRF or the abducens nucleus and ipsilateral MLF [1]. The etiologies for one-and-a-half syndrome are brain stem infarcts, demyelinating diseases (MS, ADEM), hemorrhage, aneurysm or vascular malformation; and neoplasm [2-4]. Ataxia and ophthalmoplegia occurs in CIS (clinically-isolated-syndrome) due to brain stem and cerebellar demyelination. Possible presentations of CIS are optic neuritis, a brain stem and/or cerebellar syndrome, a spinal cord syndrome, or occasionally cerebral hemispheric dysfunction $[5,6]$. Leigh disease can result from nuclear DNA mutations in genes that code for components of the respiratory chain: pyruvate dehydrogenase complex deficiency, complex I or I deficiency, complex IV (COX) deficiency, complex V (ATPase) deficiency, and deficiency of coenzyme Q10. These defects may occur sporadically or be inherited by autosomal recessive transmission, by X-linked transmission, or by maternal transmission [7]. There are focal symmetric areas of necrosis in the thalamus, basal ganglia, tegmental gray matter, periventricular and periaqueductal regions of the brainstem, and posterior columns of the spinal 
cord. Elevations in serum lactate levels are characteristic and hypertrophic cardiomyopathy, hepatic failure and rental tubular dysfunction can occur. There is no definitive treatment for the underlying disorder, but a range of vitamins including riboflavin, thiamine, and coenzyme $Q$ are often given for betterment of mitochondrial function [7].

\section{Case Presentation}

\section{Case 1}

An 8-year-old male residing at Sunderban was admitted in our hospital with chief complaints of, inability to move left upper and lower limb for 2 days with no movement of the right eye and abnormal movement of the left eye for same duration. The patient was apparently well 12 days back. The illness started with fever which was acute in onset, high grade, intermittent. Initially there was altered sensorium and drowsiness. Fever subsided and sensorium improved gradually, followed by loss of power in both left upper and lower limbs and no movement of the right eye and abnormal movement of the left eye. Fever was not associated with any rash, bleeding manifestation, headache, vomiting, and convulsion. The weakness was progressive in first $48 \mathrm{hrs}$. Later it became static and was associated with urinary retention. There was no history of tingling and numbness. Patient complained of blurring of vision to distant objects and vision improved on occluding one eye. Past history, family history, treatment history and immunization history was uneventful. Examination of neurological system revealed normal higher function. There was absence of both adduction and abduction of the right eye with loss of adduction in the left eye (right sided gaze palsy) and abduction nystagmus in the left eye. This was also associated with left hemiparesis with left sided increased tone, brisk jerks and planter extensor. Laboratory investigations showed routine blood parameters within normal limit. CSF study showed lymphocytic pleocytosis with normal protein and sugar. MRI showed areas of asymmetrical hyper intensities involving bilateral parietal areas with involvement of corpus callosum. MRI also showed areas of demyelination in brain stem (Figures 1 and 2). CSF oligoclonal bands were negative. This case was diagnosed to be ADEM with One-and-half syndrome. The patient was treated with intravenous pulse methyl prednisolone $30 \mathrm{mg} /$ $\mathrm{kg}$ intravenous for 3 days followed by oral prednisolone $2 \mathrm{mg} /$ $\mathrm{kg}$ tapered over 1 month. After treatment the patient improved dramatically. Gaze became normal in all directions and patient started walking slowly.

\section{Case 2}

A 10-year-old boy was admitted in our hospital with chief complaints of Inability to stand and sit with unsteadiness of gait for last 3 days along with double vision and abnormal movements of both eyes for the same duration. The patient was apparently well 7 days back. The illness started with fever which was acute in onset, high grade, intermittent in nature. Patient was alert and conscious with no alteration of sensorium. Fever subsided followed by unsteadiness of gait. During walking and sitting there was swaying of body to either side. Patient complained of double

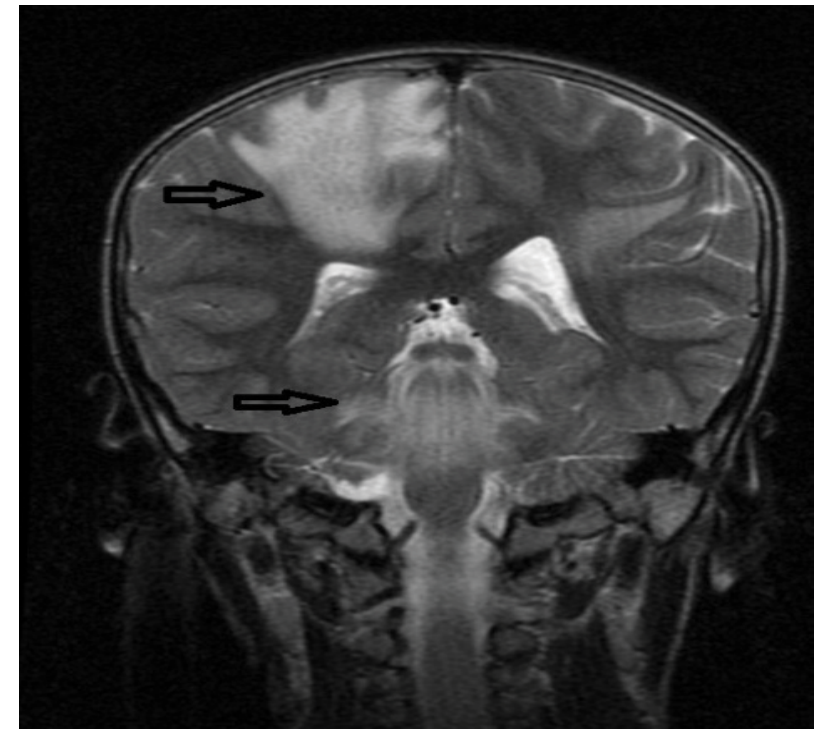

Figure 1 Showing demyelination in right parietal cortex and brain stem (black arrows).

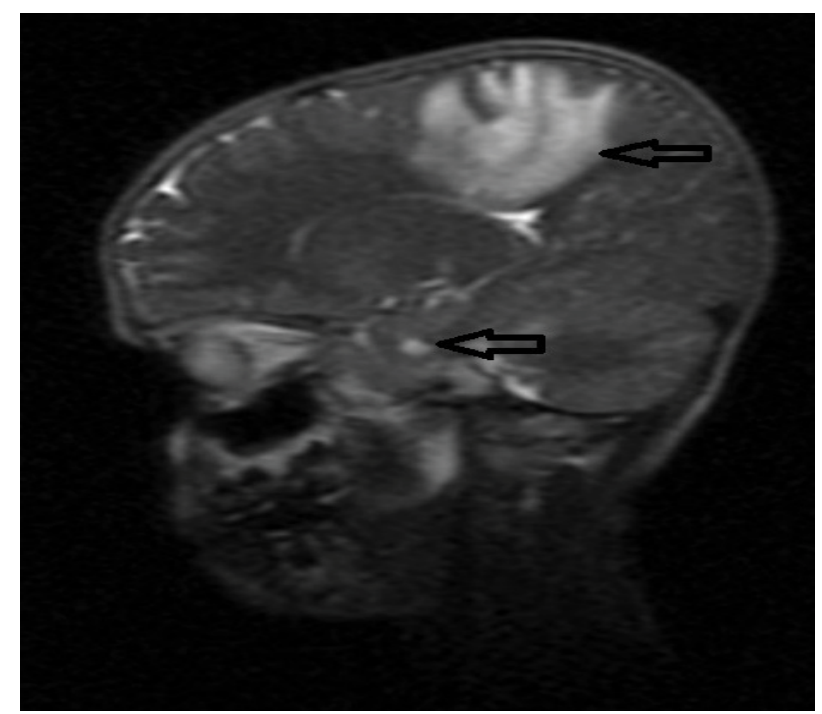

Figure 2 T2-W Sagittal section showing demyelination in cortex and brain stem (shown by black arrows).

vision to distant objects. Informant noticed abnormal movements of both eyes. Informant also noticed medial deviation of left eye and right eye was apparently centrally located. There was unsteadiness of gait and swaying of body to either side during walking and sitting without support. Patient had no bladder bowel abnormality or tingling numbness. Past history was uneventful. Neurological system examination revealed higher functions normal. There was absence of abduction in the left eye and adduction in the right eye (Left-Gaze-Palsy), Moreover, on looking towards the right there was partial loss of abduction of the right eye with nystagmus of both eyes, shown in. Deep tendon reflexes of both the limbs were exaggerated with normal power. Unsteadiness of gait was present in attempt to sit and stand without support. There was no sensory loss and other cerebellar 
signs were absent. CSF study showed a cell count 12 cells $/ \mathrm{cm}^{2}$, (lymphocytic pleocytosis), sugar $51 \mathrm{mg} / \mathrm{dl}$, total protein $25 \mathrm{mg} / \mathrm{dl}$, chloride $118 \mathrm{mg} / \mathrm{dl}$. MRI showed areas of asymmetrical hyperintensities involving bilateral sub-cortical areas of fronto-parietal region involving the frontal eye field. The demyelination of right side was more than the left (Figure 3). There was no involvement of brain stem and cerebellum. CSF oligoclonal bands were absent. Fundoscopy, VEP (Visual Evoked Potential), BERA (brainstem evoked response audiometry), NCV (nerve conduction velocity) all were normal. ANA and anti NMDA receptor antibodies were negative ruling out autoimmune encephalitis. The patient was treated with intravenous pulse methyl-prednisolone $30 \mathrm{mg} / \mathrm{kg}$ for 3 days followed by oral prednisolone $2 \mathrm{mg}$ per $\mathrm{kg}$ tapered over 1 month. After treatment the patient improved dramatically and never had recurrences. Follow up MRI was done at 3 months and came to be normal. Patient is still in follow up 6 monthly for a period of 2 years and absolutely normal with no further recurrences. This case was diagnosed to be CIS with ataxia and ophthalmoplegia due to hemispheric dysfunction with brain stem and cerebellum being normal, a rare entity.

\section{Case 3}

An 18-month-old male child, a product of non- consanguineous marriage, presented with chief complaints of delayed developmental milestones and frequent falls during walking. Inability to move the eyeballs with absent conjugate eye movements in all directions. The child was born by normal vaginal delivery and cried immediately after birth. Weight was not recorded. Child was exclusively breast fed and weaning was done with rice, mashed potato etc. Mother noticed less movement (apparently fixed) of the eyeballs (Figure 4) since 6 month of age. Child achieved neck holding at 6 month, sitting with support at 9 month and standing with support at 16 month and now at 18 month he walks with support with broad based gait and falls frequently. Laboratory investigations showed Routine blood tests were normal. Blood lactate level was raised (venous blood). CSF lactate: normal. MRI Showed T2W hyper intense lesions in brainstem and cerebellar peduncles and also hyper intense lesions at brainstem region in DW images (? Infarct/necrosis) (Figures 5-7). After 5 months follow up MRI was repeated and it revealed

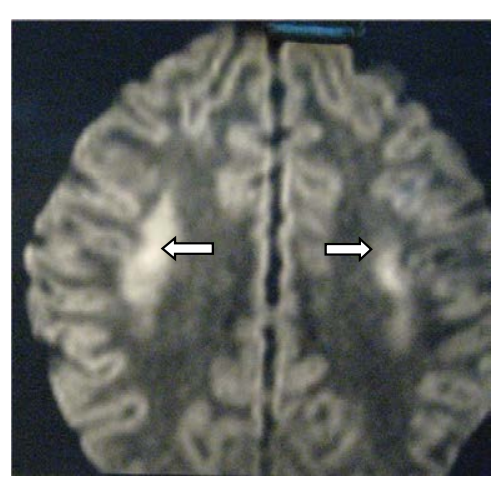

Figure 3 Asymmetrical hyperintensities in bilateral subcortical areas with right side affected more than left (marked by white arrowheads).
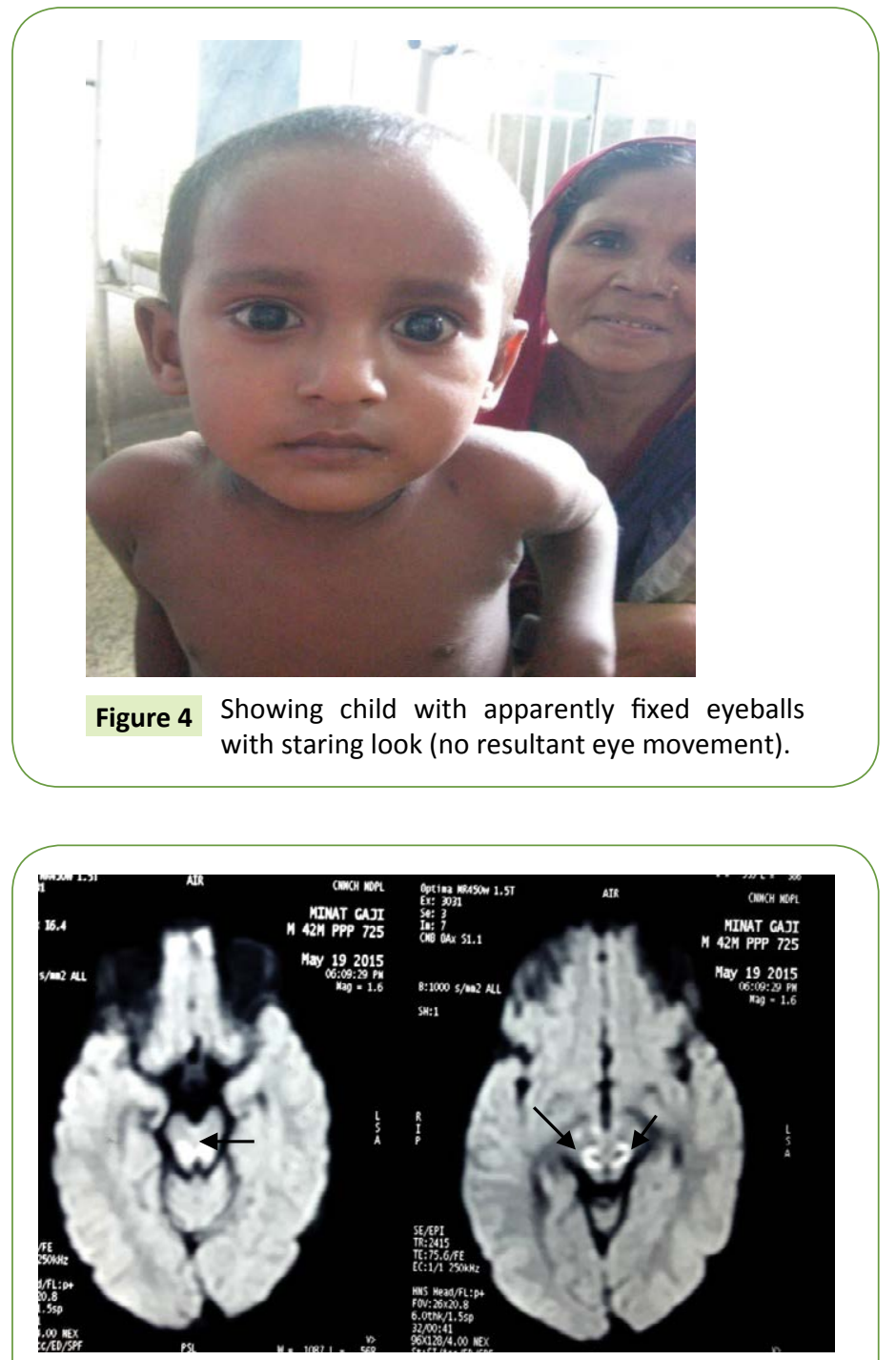

Figure 5 Diffusion weighted DW images showing hyper intense lesions at brain stem (black arrows).

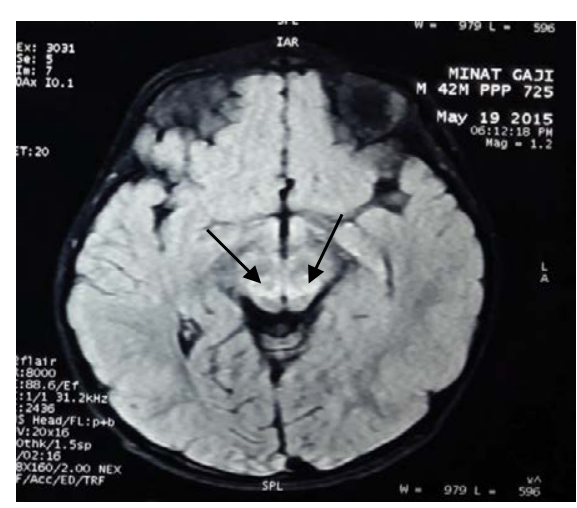

Figure 6 T1 FLAIR axial image showing hyperintensity with a small central hypo-intense area at brainstem (? Infarct/necrosis) (black arrows).

new areas of increased signal intensities in both the thalami (Figure 8) and MR spectroscopy revealed double lactate peak (Figure 9) consistent with sub-acute necrotizing mitochondrial 


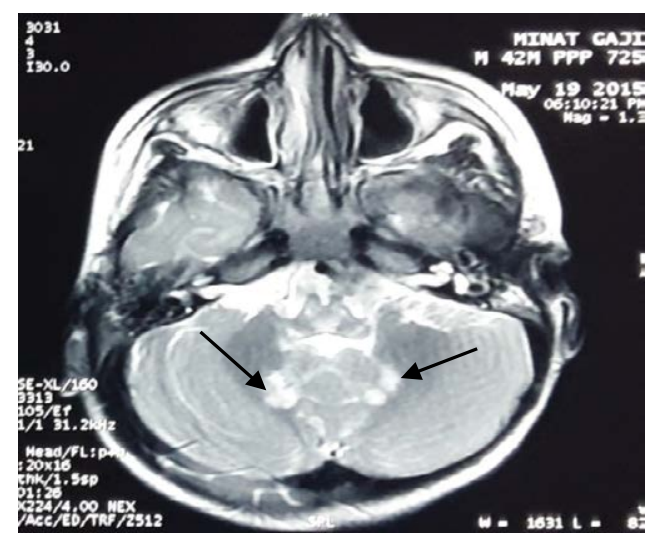

Figure 7 Axial T2 W image shows hyper intensities at cerebellar peduncles. (black arrows).

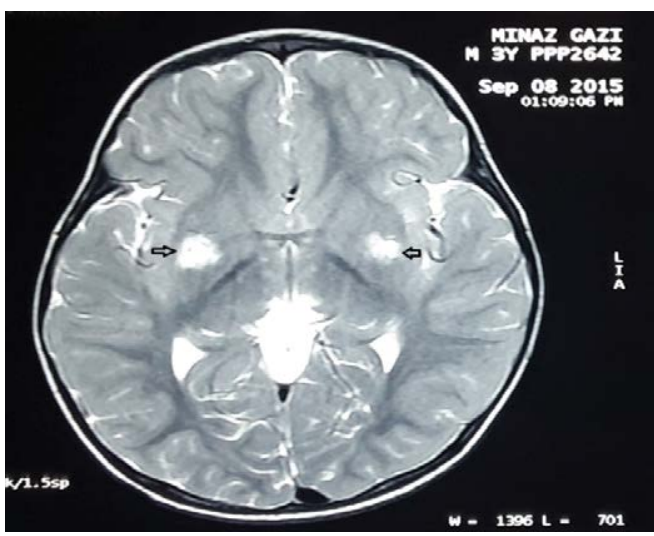

Figure 8 Shows bilateral T2 W hyper intense lesions in both the thalami (black arrows).

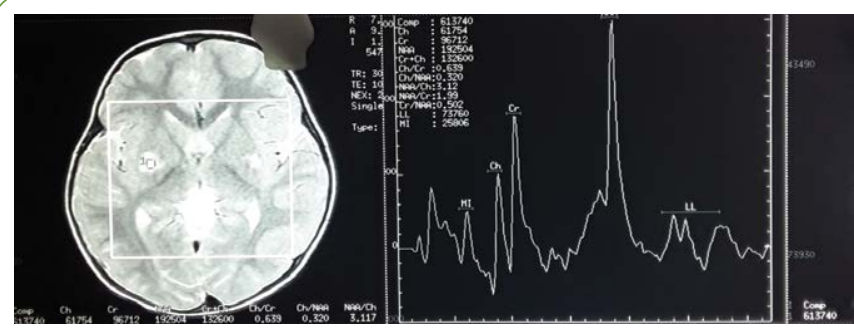

Figure 9 Double Lactate peak in MR Spectroscopy from the hyper intense lesion of thalamus.

encephalomyopathy (Leigh disease). Though ophthalmoplegia is seen frequently in mitochondrial diseases like KSS (Kearn-Sayre Syndrome), but complete loss of conjugate movements of both the eyes is a rare entity in Leigh disease so far reported.

\section{Discussion}

One and half syndrome is characterized by lateral gaze palsy along with internuclear opthalmoplegia [8]. The main causes of this rare syndrome are stroke and multiple sclerosis [1]. The common signs of ADEM include visual loss, cranial neuropathies, ataxia, motor and sensory deficits with bladder bowel dysfunction in concurrent spinal cord involvement [9]. But one and half syndrome is a very rare finding in ADEM, so far reported. The lesion is located in the para pontine reticular formation (PPRF), 6th nerve nucleus, (Figure 10) and medial longitudinal fasciculus (MLF) $[10,11]$ as shown by demyelination in the right brain stem region. Thus one should not miss the eye in ADEM. Clinically isolated syndrome (CIS) is a central nervous system demyelinating event isolated in time and there is every chance of future development of multiple sclerosis (MS) [5]. Magnetic resonance imaging (MRI) is currently the most useful tool to evaluate risk. Cerebrospinal fluid studies with presence of oligoclonal bands may also be used to assess the likelihood of MS [12]. Useful radiographic predictors of MS include the presence of multifocal homogenous or ring-enhancing white matter foci, as well as T2hyperintense lesions affecting the corpus callosum $[13,14]$ or the poster lateral compartment of the spinal cord [15]. However, not everyone who experiences a clinically isolated syndrome will go on to develop MS. According to the site of demyelination in brain, the symptoms of CIS vary. But gaze palsy without brain stem involvement and unsteadiness of gait without cerebellum is a rare phenomenon in medical literature. In our case there is involvement of frontal eye field fibers controlling the lateral gaze after crossing at mid brain level and ending in opposite 6 th nerve nucleus, which is further connected to contralateral $3^{\text {rd }}$ nerve nucleus through Medial longitudinal fasciculus (MLF) (Figure 11). As there is more involvement of right sub cortical region than the left, there is left gaze palsy and only partial abduction failure at right gaze [16]. The unsteadiness of gait can be explained by the involvement of fronto-ponto- cerebellar fibers in this case. But in the absence of other cerebellar signs, the swaying movement of the body should better be explained by the ophthalmoplegia giving rise to diplopia and thus disrupting the visual pathway maintaining body equilibrium. Leigh disease is a progressive degenerative disorder presenting in infancy.

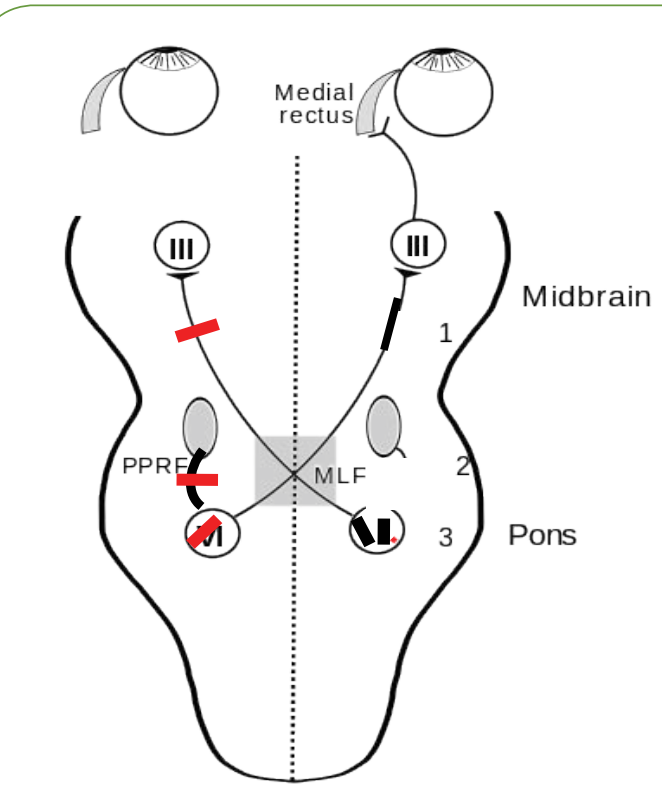

Figure 10 Involvement of right sided PPRF (2), $6^{\text {th }}$ nerve nucleus (3) and MLF (1) thus affecting right lateral gaze and adduction of left eye (One-and-half syndrome). 


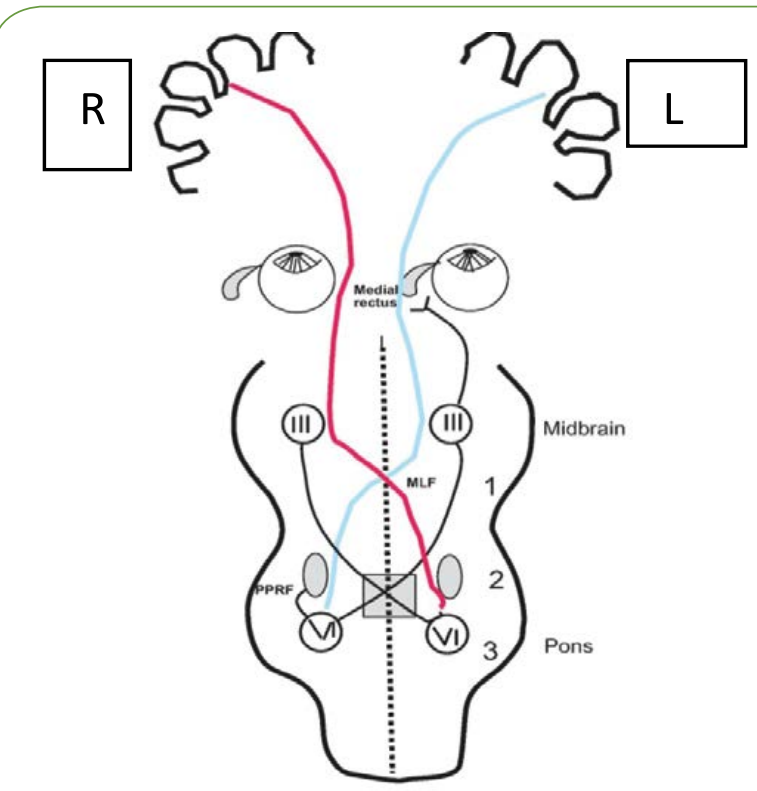

Figure 11 Frontal eye field fibers controlling lateral gaze (1 denotes IIIrd nerve nucleus at midbrain, 2 denotes PPRF, i.e., parapontine reticular formation, 3 denotes $\mathrm{VI}^{\text {th }}$ nerve nucleus at the level of pons).

Presents with feeding and swallowing problems, vomiting, and failure to thrive associated with lactic acidosis. Lesions seen in the brainstem and/or basal ganglia on MRI. Approximately $30 \%$ of cases are caused by mutations in mtDNA. Delayed motor and language milestones may be evident, and generalized

\section{References}

1 De SJ, Lucas C, Leclerc X, Sahli A, Vermersch P, et al. (1999) One-anda-half syndrome in pontine infarcts: MRI correlates. Neuroradiology 41: 666-669.

2 Kim JS (2004) Internuclear ophthalmoplegia as an isolated or predominant symptom of brainstem infarction. Neurology 62: 14911496.

3 Anderson CA, Sandberg E, Filley CM, Harris SL, Tyler KL (1999) One and one-half syndrome with supranuclear facial weakness: magnetic resonance imaging localization. Arch Neurol 56: 1509 -1511.

4 Zee DS (1992) Internuclear ophthalmoplegia: Pathophysiology and diagnosis. Baillieres Clin Neurol 1: 455-470.

5 Miller D, Montalban B (2005) Clinically isolated syndromes suggestive of multiple sclerosis, part 1: Natural history, pathogenesis, diagnosis and prognosis. Lancet Neurol 4: 281-288.

6 Frohman EM (2003) Multiple sclerosis. Med Clin North Am 87: 867-897.

7 Michael VJ (2016) Mitochondrial Encephalomyopathies in Kliegman. Stanton, St. Geme, Schor, Behrman, Nelson Textbook of Paediatrics (20th edn), Philadelphia Saunders Elsevier pp: 2902.

8 Mazumdar J, Ghosh KC, Das PK (2014) Do not miss the eye in acute demyelinating encephalomyelitis. Indian J Pediatr 81: 1427-1428. seizures, weakness, hypotonia, ataxia, tremor, pyramidal signs, and nystagmus are prominent findings [7]. Abnormal results on CT or MRI scan consist of bilaterally symmetric areas of low attenuation in the basal ganglia and brainstem as well as elevated lactic acid on MR spectroscopy [7]. Among the mitochondrial encephalomyopathies, Ophthalmoplegia is frequently seen in KSS (Kearns- sayre syndrome) but Leigh disease with complete ophthalmoplegia (absence of eye movement in all directions) is very rare as per medical literature till date.

\section{Conclusion}

So from the above case studies and discussion we can draw a conclusion that eye movement abnormality rather gaze palsy in children may have a various etiologies underneath. So as a clinician we have to rule out the etiologies to reach a diagnosis with the help of history, clinical findings, and investigations. MRI now a day is of great help in diagnosing the demyelination underlying.

\section{Acknowledgements}

I am thankful to my head of the department, pediatrics, Dr. P.K. Das (Calcutta National Medical College and Hospital) and all my co-authors of the published articles. I am also very much thankful to the author Yun Jung Bae, MD for the immense support that I have got from his article "Brainstem Pathways for Horizontal Eye Movement: Pathologic correlation with MR imaging" (Radiographic).

9 Jayne MN (2016) Demyelinating disorders of central nervous system. Kliegman. Stanton, St. Geme, Schor, Behrman, Nelson Textbook of Paediatrics (20th edn), Philadelphia Saunders Elsevier pp: 2920.

10 Bae YJ, Kim JH, Choi BS, Jung C, Kim E (2013) Brainstem pathways for horizontal eye movement: Pathologic correlation with MR imaging. Radiographics 33: 47-59.

11 Thömke F, Hopf HC, Krämer G (1992) Inter-nuclear ophthalmoplegia of abduction: clinical and electrophysiological data on the existence of an abduction paresis of pre-nuclear origin. J Neurol Neurosurg Psychiatry 55:105-111.

12 Marcus JF, Waubant EL (2013) Updates on clinically isolated syndrome and diagnostic criteria for multiple sclerosis. Neurohospitalist 3: 65-80.

13 Bakshi R, Minagar A, Jaisani Z, Wolinsky JS (2005) Imaging of multiple sclerosis: role in neurotherapeutics. NeuroRx 2: 277-303.

14 Zivadinov R, Cox JL (2007) Neuroimaging in multiple sclerosis. Int Rev Neurobiol 79: 449-474.

15 Cordonnier C, De SJ, Breteau G, Ferriby D, Michelin E, et al. (2003) Prospective study of patients presenting with acute partial transverse myelopathy. J Neurol 250: 1447-1452.

16 Mazumdar J, Kanjilal S, Ghosh KC (2015) Clinically isolated syndrome (CIS) presenting with ataxia and ophthalmoplegia. J Mult Scler 2: 0389.1000155. 